Draft Version February 23, 2021

Typeset using LATEX twocolumn style in AASTeX63

\title{
A self-consistent simulation of proton acceleration and transport near a high-speed solar wind stream
}

\author{
Nicolas Wijsen (D), ${ }^{1}$ Evangelia Samara (D), ${ }^{2,1}$ Àngels Aran (D), ${ }^{3}$ David Lario (D), 4 Jens Pomoell (D), ${ }^{5}$ And \\ StefaAn Poedts (iD 1,6
}

\author{
${ }^{1}$ Centre for mathematical Plasma-Astrophysics, Department of Mathematics, KU Leuven, Celestijnenlaan 200B, B-3001 Leuven, Belgium \\ ${ }^{2}$ Royal Observatory of Belgium, Brussels, Belgium \\ ${ }^{3}$ Department of Quantum Physics and Astrophysics, Institute of Cosmos Sciences (ICCUB), Universitat de Barcelona (UB-IEEC), Spain \\ ${ }^{4}$ NASA, Goddard Space Flight Center, Heliophysics Science Division, USA \\ ${ }^{5}$ Department of Physics, University of Helsinki, Helsinki, Finland \\ ${ }^{6}$ Institute of Physics, University of Maria Curie-Sktodowska, Lublin, Poland
}

(Received January 13, 2021; Revised February 1, 2021; Accepted February 1, 2021)

Submitted to ApJ lett.

\begin{abstract}
Solar wind stream interaction regions (SIRs) are often characterised by energetic ion enhancements. The mechanisms accelerating these particles, as well as the locations where the acceleration occurs, remain debated. Here, we report the findings of a simulation of a SIR event observed by Parker Solar Probe at $\sim 0.56$ au and the Solar Terrestrial Relations Observatory-Ahead at $\sim 0.95$ au in September 2019 when both spacecraft were approximately radially aligned with the Sun. The simulation reproduces the solar wind configuration and the energetic particle enhancements observed by both spacecraft. Our results show that the energetic particles are produced at the compression waves associated with the SIR and that the suprathermal tail of the solar wind is a good candidate to provide the seed population for particle acceleration. The simulation confirms that the acceleration process does not require shock waves and can already commence within Earth's orbit, with an energy dependence on the precise location where particles are accelerated. The three-dimensional configuration of the solar wind streams strongly modulates the energetic particle distributions, illustrating the necessity of advanced models to understand these particle events.
\end{abstract}

Keywords: Solar wind (1534) — Corotating streams (314) — Interplanetary particle acceleration (826)

\section{INTRODUCTION}

The interaction of a high-speed solar wind stream (HSS) with slower solar wind ahead results in the formation of a stream interaction region (SIR; Gosling \& Pizzo 1999). SIRs observed at 1 au are usually bounded by forward and reverse compression waves (FCW and RCW). The FCW accelerates and compresses the slow solar wind propagating in front of the HSS, whereas the RCW decelerates and compresses the fast solar wind of the HSS. At larger heliocentric distances, such pres-

Corresponding author: Nicolas Wijsen

nicolas.wijsen@kuleuven.be sure waves commonly steepen into forward and reverse shocks (FS and RS) (Gosling \& Pizzo 1999).

It was originally suggested that recurrent energetic ion intensity enhancements seen at 1 au in association with HSSs were produced by these outer forward-reverse shock pairs (e.g., Barnes \& Simpson 1976; Fisk \& Lee 1980). Intensity radial gradients (Van Hollebeke et al. 1978) and flow directions (Marshall \& Stone 1978) of the SIR-associated energetic ions were consistent with the origin of the particles at several au (Mason \& Sanderson 1999). However, Giacalone et al. (2002) realised that compression waves can already induce a first-order Fermi acceleration process, without needing to steepen into shock waves. This happens when the spatial extent of the compression is significantly smaller than the mean free path of the particles across the wave, such that the 
particles experience the compression wave much like a shock (Giacalone et al. 2002). In addition, a secondorder Fermi acceleration process may also contribute to the local ion acceleration in SIRs (e.g., Richardson 1985; Schwadron et al. 1996, 2020).

An important question remains with regard to the dominant source of the SIR-associated energetic ions detected at $1 \mathrm{au}$, namely whether they originate from distant shock waves or from close-by compression waves. Observations from Helios (Porsche 1975), Parker Solar Probe (PSP; Fox et al. 2016), and Solar Orbiter (SolO; Müller et al. 2013) have shown that SIR energetic ions are detected well within the Earth's orbit (e.g., Van Hollebeke et al. 1978; McComas et al. 2019; Desai et al. 2020; Cohen et al. 2020; Allen et al. 2020a,b,c; Joyce et al. 2020; Schwadron et al. 2020). Hence, the study and modelling of SIR particle events at different heliocentric distances is essential in order to address the acceleration and transport of energetic particles.

In this work, we study a SIR event observed (Allen et al. 2021) in September 2019 by both PSP (located at $\sim 0.56 \mathrm{au}$ ) and the Solar Terrestrial Relations Observatory-Ahead (STEREO-A) spacecraft (located at $\sim 0.95 \mathrm{au}$ ). The close radial alignment between STEREO-A and PSP at that time makes this event especially interesting because the evolution of the coronal hole responsible for the HSS observed by both spacecraft is minimal (Allen et al. 2021). To understand this SIR event in depth, a realistic scale-bridging model of the solar wind and energetic particle acceleration and transport is necessary. In this work, this is achieved by using the combination of two novel models, namely, the EUropean Heliospheric FORcasting Information Asset (EUHFORIA; Pomoell \& Poedts 2018) and the PArticle Radiation Asset Directed at Interplanetary Space Exploration (PARADISE; Wijsen et al. 2019a; Wijsen 2020). EUHFORIA is a data-driven magnetohydrodynamic (MHD) model of the solar wind, whereas PARADISE simulates energetic particle distributions in the solar wind provided by EUHFORIA, using a quasi-linear (Jokipii 1966) approach to capture the interaction between solar wind turbulence and energetic particles. We illustrate how EUHFORIA+PARADISE captures, in a self-consistent manner: (1) the complex HSS structure, (2) the formation of the SIR, (3) the acceleration of protons at both the FCW and the RCW, and (4) the propagation of the energetic protons into the inner heliosphere. We show how the spatial distribution of SIR particles varies as the compression waves tend to steepen at large helioradii. Whereas low-energy particles $(\lesssim 0.2$ $\mathrm{MeV}$ ) can be produced at small helioradii, the acceler- ation of more energetic particles requires steeper compression waves.

\section{RESULTS}

\subsection{The Solar Wind}

Figure 1 shows the solar wind bulk speed, proton number density, and magnetic field magnitude observed by PSP (left) and STEREO-A (right). The gaps in the PSP data correspond to time periods when the instruments on board the spacecraft were powered off. The results of the data-driven EUHFORIA solar wind simulation are shown in red. The precise As can be seen, the EUHFORIA simulation succeeds at reproducing the observed speed profile at STEREO-A, with the simulation capturing the amplitude of the HSS as well as the decreasing speed profile in the rarefaction region behind the HSS. Reproducing the speed profile of the solar wind is essential when studying energetic particle populations. This is because the frozen-in condition of the solar wind magnetic field implies that the large-scale magnetic field connectivity is largely dominated by the solar wind plasma flow.

The arrival of the HSS at PSP occurred in the middle of a data gap (Fig. 1a). The EUHFORIA model (red solid line) predicts a solar wind speed increase more gradual than what the available PSP data suggests. This simulated gradual increase translates into a small magnetic field increase at the SIR in comparison with the observations (cf. Fig. 1e). STEREOA data suggests the presence of a developing RS on 22 Sep 01:35 UT (Allen et al. 2021) as shown by the abrupt decrease in magnetic field magnitude. Allen et al. (2021) found that PSP solar wind data aligns well with STEREO-A data when the latter is shifted 1.77 days in time to account for corotation. The dashed line in Fig. 1a shows the simulated speed profile observed by STEREO-A, shifted 1.77 days earlier in time. For such a more abrupt speed increase, the maximum speed at PSP would be obtained $18 \mathrm{~h}$ earlier than in the current simulation. The gradual speed increase at PSP in the simulation results partly from an overestimation of the solar wind speed just ahead of the HSS (see Fig. 1a). The existence of a strong speed gradient in the solar wind at 0.5 au suggests a very abrupt transition between the slow and fast solar wind source regions.

Figure 2 displays the three-dimensional structure of the HSS. Figure 2a shows the solar wind speed $V_{\mathrm{sw}}$ at the constant latitude $\vartheta=2.3^{\circ}$ (using the Heliocentric Earth Equatorial (HEEQ) coordinate system), which is approximately the latitude of PSP and STEREO-A during the SIR passage. The symbols indicate the location of STEREO-A (black dots) and PSP (grey triangles) 

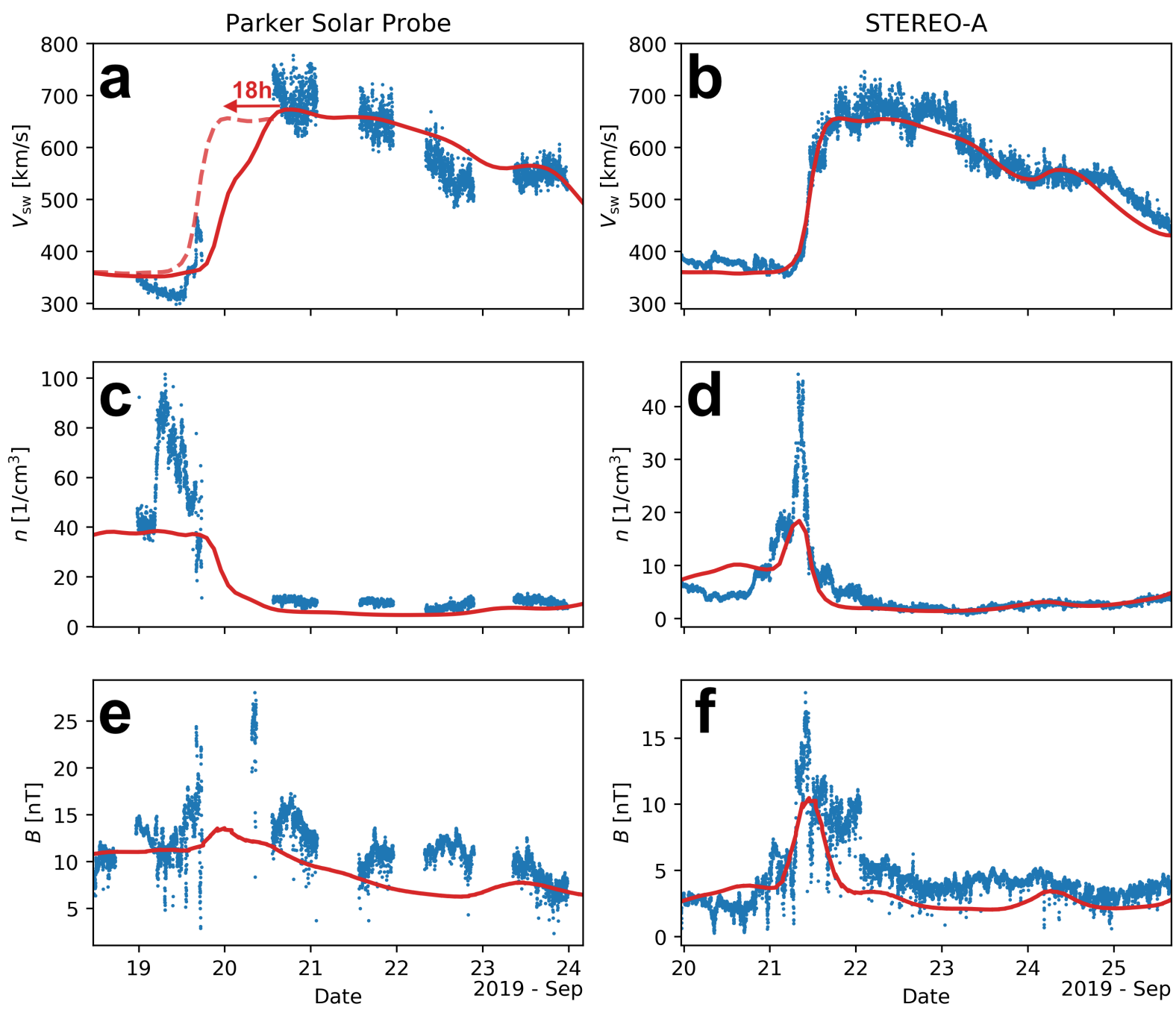

Figure 1. Comparison between the proton solar wind speed ( $a-b)$, density $(c-d)$, and magnetic field magnitude (e-f) observed (blue dots) by PSP (left) and STEREO-A (right) with the EUHFORIA simulation results (red solid lines). The dashed line in panel (a) shows the simulated HSS onset observed at STEREO-A shifted earlier in time by 1.77 days (Allen et al. 2021) to account for corotation.

at different times. The black lines are interplanetary magnetic field (IMF) lines crossing the spacecraft and projected on the $\vartheta=2.3^{\circ}$ plane. The apparent crossing of these IMF lines inside the SIR is solely a projection effect. The non-zero latitudinal component of the IMF arises mainly due to the deflection of the magnetic field at the FCW and the RCW (Wijsen et al. 2019b).

Figure 2c shows $V_{\mathrm{sw}}$ at a constant helioradius $r=$ 1.5 au. The HSS appears as a southward extension of the fast solar wind originating from the northern polar coronal hole. The magnetic connection of PSP and STEREO-A at 1.5 au is indicated by the grey triangles and the black dots and at the same times as in Fig. 2a. Both spacecraft connect to similar regions of the SIR, yet for a different time duration. PSP has a magnetic connection within the SIR at 1.5 au for approximately three full days (19 Sep 12:00 to 21 Sep 12:00), whereas STEREO-A is only connected for $\sim 1.5$ days (20 Sep 12:00 to 22 Sep 00:00) to the same region.

Figures $2 \mathrm{~b}$ and $2 \mathrm{~d}$ show the divergence of the solar wind velocity $\nabla \cdot \mathbf{V}_{\mathrm{sw}}$, which is a measure of the local plasma compression. Most of the solar wind is in an expanding state, characterised by $\nabla \cdot \mathbf{V}_{\mathrm{sw}}>0$. These are the regions where particles undergo adiabatic cooling while propagating (Ruffolo 1995). In contrast, the FCW and the RCW bounding the SIR are regions where $\nabla \cdot \mathbf{V}_{\mathrm{sw}}<0$. Both waves are clearly visible in Fig. $2 \mathrm{~b}$ as two spiral-shaped blue regions, with larger negative values of $\nabla \cdot \mathbf{V}_{\mathrm{sw}}$ at the RCW. Figure $2 \mathrm{~d}$ illustrates the complex latitudinal structure of the SIR, showing that the solar wind contains several additional compressed plasma regions that are all characterised by a 

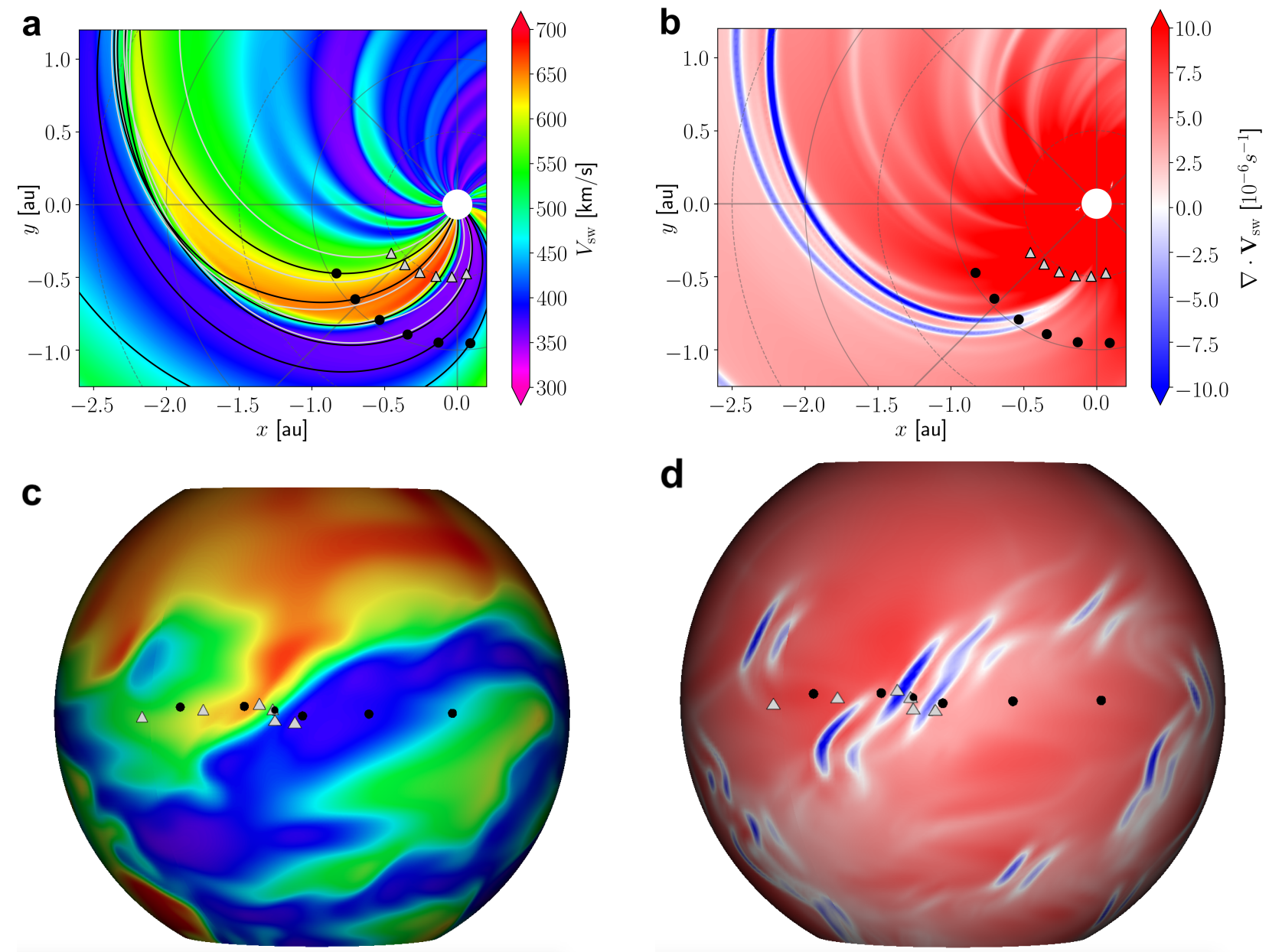

Figure 2. Simulated solar wind speed (panels a and c) and the divergence of the solar wind velocity (panels b and d) at HEEQ latitude $\vartheta=2.3^{\circ}$ (upper row) and at the radial distance $r=1.5$ au (lower row). The symbols in panels a and $\mathrm{b}$ represent the location of STEREO-A (black dots) and PSP (grey triangles) at a 24h cadence, ranging from 18 Sep 12:00 UT to 23 Sep 12:00 UT (from right to left). The dots and triangles in c and d represent the corresponding magnetic connection of STEREO-A and PSP, respectively

clear two-component structure, that is, a FCW followed by a (stronger) RCW. Each of these compression regions forms a potential source of accelerated particles. However, most of these regions are located well above or below the ecliptic. Figure 2d shows that, after passing the RCW, the magnetic connection of both STEREO-A and PSP skim the northern edge of another RCW, associated with the southern edge of the HSS. As discussed below, this second RCW may be responsible for the small particle intensity enhancements seen at STEREO-A on 23 Sep (see Fig. 3).

\subsection{Energetic Particles at STEREO-A}

Figure 3a shows the 10 minute averaged ion intensities observed by the Solar Electron and Proton Telescope (SEPT; Müller-Mellin et al. 2008) on board STEREO$\mathrm{A}$ in four different energy channels, after subtracting the corresponding pre-event intensity values (averaged from Sep 18 00:00 UT to Sep 19 12:00 UT). Intensity enhancements at energies below $\sim 200 \mathrm{keV}$ were observed already on Sep 20 09:00 UT, whereas $\gtrsim 500 \mathrm{keV}$ proton intensities did not increase until mid 21 Sep. Low-energy $(\lesssim 500 \mathrm{keV})$ proton intensities continued to be elevated until 23 Sep 12:00 UT.

To simulate these particle populations, a seed population of $40 \mathrm{keV}$ protons is injected into both the RCW and the FCW bounding the SIR. The PARADISE model propagates these protons through the EUHFORIA solar wind, taking into account the effect of small-scale solar wind turbulence on the energetic particles. The latter is done using results from quasi-linear theory, which translates the scattering processes of particles by magnetic fluctuations into an anisotropic pitch-angle diffusion process in the reference frame co-moving with the solar wind (Jokipii 1966). This diffusion process drives the particle acceleration in the solar wind regions where 

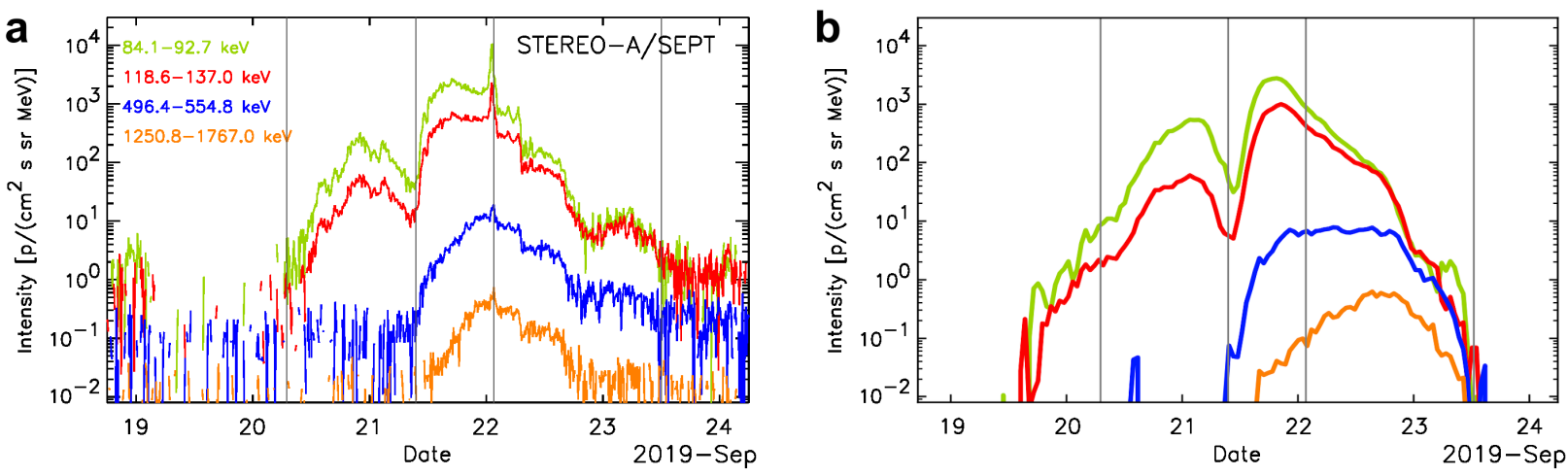

Figure 3. Observed (left) and simulated (right) omnidirectional ion intensities at STEREO-A. The vertical lines indicate the onset time of the SIR event (Sep 20 09:00 UT), the SI (Sep 21 09:30 UT), the developing RS (22 Sep 01:35 UT), and the stop time of the SIR event (23 Sep 12:00 UT).

$\nabla \cdot \mathbf{V}_{\mathrm{sw}}<0$, as it models the interaction of particles with converging scattering centres (e.g., le Roux \& Webb 2012; Zank 2014). In addition to pitch-angle diffusion, a weak spatial diffusion process perpendicular to the IMF is considered. The precise set-up of the PARADISE simulation is described in Appendix B.

Figure $3 \mathrm{~b}$ shows the simulated intensity profiles, under the assumption that the protons propagate with constant mean free paths along and across the IMF of $\lambda_{\|}=0.3 \mathrm{au}$ and $\lambda_{\perp}=10^{-4}$ au, respectively. The assumption that $\lambda_{\perp} / \lambda_{\|} \sim 10^{-3}$ implies that the energetic particles are predominantly propagating along the IMF lines. Figure $3 \mathrm{~b}$ shows that the PARADISE simulation successfully reproduces several features seen in Figure 3a, specifically: (1) the onset and ending times of the SIR event; (2) the double-peaked structure of the intensity-time profiles, with the second peak showing the highest intensities; (3) the soft energy spectrum of the first peak compared to the second peak, with the first peak showing very few protons above $\sim 500 \mathrm{keV}$; (4) the sudden strong increase in the particle intensities of the two lowest energy channels around 21 Sep 10:00 UT before reaching a second peak; (5) the gradual increase in the two high-energy channels, hence producing an overall energy spectrum that hardens with the passing of the SIR; (6) the fact that intensity increases are being observed at $\mathrm{MeV}$ energies; and (7) the energy spectrum obtained from integrating the intensity over the entire event that has a power-law dependence of $E^{-2.94 \pm 0.01}$ in the data and $E^{-2.90 \pm 0.02}$ in the simulation. Here, $E$ denotes the particle energy and the power-law fit was performed for the energy range $84.1-1985.3 \mathrm{keV}$.

The double-peaked profile is a result of the particles being accelerated at either the FCW or the RCW. The FCW is weaker than the RCW, and hence less efficient in accelerating particles to high energies. Because of the $\nabla \cdot \mathbf{V}_{\mathrm{sw}}$ configuration (Figures $2 \mathrm{~b}$ ), particle accelera- tion occurs from $\sim 0.8$ au onward, with the most efficient particle acceleration occurring around $\sim 1.5$ au. As a result, the majority of the accelerated protons detected at STEREO-A in the simulation are observed to be streaming in the sunward direction, even though some low-energy protons $(\lesssim 90 \mathrm{keV})$ are already accelerated within 1 au. In addition, since our solar wind simulation underestimates the speed gradient at the location of PSP, it is very likely that, in reality, more particles were already accelerated within 0.8 au.

The observed particle intensities show a sudden spike on 22 Sep 01:35 UT, which coincides with the passage of the developing RS. However, in the EUHFORIA simulation, no shock wave is present as the RCW is still steepening beyond 0.95 au. Only at radial distances beyond 1 au does the RCW steepens enough to efficiently accelerate particles to $\mathrm{MeV}$ energies. This explains why, in the simulation, the $\mathrm{MeV}$ energy channel peaks too late compared to the data. In contrast, the peak intensities of the two lowest energy channels in Fig. 3b coincides with the passage of the simulated FSW and the RSW, indicating that these particles are accelerated locally.

In the simulation, the widths of the compression regions remain larger than the widths of the observed transitions due to the size of the EUHFORIA computational grid. However, the diffusive length scale of the particles across the compression waves is, in the simulation, substantially larger than the width of the compression waves itself. As a result, particles accelerate similarly to a diffusive shock acceleration process (Giacalone et al. 2002; Wijsen et al. 2019a). The good agreement between the simulated and observed energy spectra, confirms that shock waves are not required for producing energetic particle enhancements near SIRs.

The intensity dip observed around Sep 21 09:30 UT coincides with the stream interface (SI) of the SIR, that is, the region inside the SIR where the compressed fast so- 
lar wind meets the compressed slow solar wind (Gosling et al. 1978). The SI separates the two energetic particle populations that are accelerated at the FCW and the RCW. The IMF lines directly adjacent to the SI are located inside the developing SIR from small radial distances onward, and as a result, they never cross the FCW or the RCW where particles may accelerate. In a simulation with zero cross-field diffusion and no particle drifts, the suprathermal particle intensity would thus drop to zero at the SI. In contrast, if the cross-field diffusion would be more efficient, the two intensity peaks would merge into a single enveloping peak (Richardson 1985; Wijsen et al. 2019c). Since this is not the case in the observed intensity profiles, strong cross-field diffusion near the SI is inconsistent with the observations.

The observed intensities show a sudden discontinuity on 22 Sep 7:00 UT, which coincides with a rotation in the magnetic field vector (not shown here). This smallscale structure is not resolved by EUHFORIA and hence the same is true for PARADISE.

Finally, Fig. 3a shows that the particle intensities in the two lowest-energy channels had an additional small third increase on 23 Sep 01:00 UT. In the simulation, this date corresponds to the time when the magnetic connection of STEREO-A is skimming the northern edge of the second RCW shown in Fig. 2.

\subsection{Energetic Particles at PSP}

Figure 4 shows the simulated particle intensities at PSP (red line) together with the count rate of energetic ions measured by the Time-of-Flight (ToF) system of the EPI-Lo instrument (McComas et al. 2016) on board PSP (blue dots). We use hourly averages accumulated over all the apertures of EPI-Lo and consider the energy ranges provided in the NASA Space Physics Data Facility $^{1}$. EPI-Lo data was only available for those periods plotted in Figure 4, suggesting that intensity enhancements were present between 19 Sep 14:00 UT and 21 Sep 23:30 UT.

In our simulation, the omnidirectional intensities at PSP peak around 21 Sep 00:00 UT, which does not agree with the decreasing trend of the count rates observed at that time. However, we recall that, in the solar wind simulation, the transition from the slow to fast solar wind is inferred to be too smooth at PSP. In particular, the time difference between the peaks of the dashed and solid lines in Fig.1a is $\sim 18 \mathrm{~h}$. Figure 4 shows that by shifting the simulated particle intensity profiles by $18 \mathrm{~h}$ (dashed red line), a qualitative agreement is obtained between the simulation and the EPI-Lo data, both at

${ }^{1}$ cdaweb.gsfc.nasa.gov the event onset and the decay phase. This further confirms that the solar wind speed gradient at PSP most likely was nearly as steep as the one observed at STA. Such a steep speed gradient indicates that particle acceleration may already have been occurring inside 0.5 au. However, the shifted curve in Fig. 4 shows a first peak on Sep 18 associated with the FCW, suggesting that this enhancement was likely below the background level of the instrument, and indicating that the acceleration process at the FCW was not efficient at $\sim 0.5$ au.

\subsection{Three-dimensional Proton Distributions}

Figure 5 shows the omnidirectional particle intensity on spherical shells located at $0.5 \mathrm{au}$ (top row) and $1.5 \mathrm{au}$ (bottom row) for the energy channels $84.1-92.7 \mathrm{keV}$ (left) and 496.4 - $554.8 \mathrm{keV}$ (right). These figures show how the spatial dependence of the particle population is strongly modulated by the shape of the HSS. Whereas $\sim 0.5 \mathrm{MeV}$ particles are mostly found within the HSS, the $\sim 80 \mathrm{keV}$ particles extend also to the slower solar wind. At low energies, the maximum intensities at $1.5 \mathrm{au}$ are centred around the $\mathrm{RCW}$ and $\mathrm{FCW}$, whereas at $0.5 \mathrm{au}$ the particles are more dispersed and two intensity peaks are attained within the slow solar wind in front of the FCW and in the fast solar wind stream behind the RCW. Such a spatial dependence is often observed in SIR events (see Richardson 2018, and references therein) and is a consequence of nonlocal particle acceleration.

Figure 5 also illustrates how the magnetic connection of PSP (red triangles) only crosses the southward edge of the intensity peak associated with the FCW in the simulation. If the spacecraft had been located at slightly higher latitude, it would have observed significantly higher particle intensities at the FCW, according to the simulation. This might further explain why PSP did not see any particle enhancement associated with the FCW (Figure 4). The strong latitudinal dependence of the particle distribution follows from the intricate structure of the fast solar wind stream (Figure 2c). This illustrates that two spacecraft located at slightly different latitudes can see very different particle patterns. This finding is in agreement with the results of Jian et al. (2019), who analysed 151 pairs of SIRs seen by STEREO A and B and showed that, even within $5^{\circ}$ of latitude, the solar wind properties of a single SIR can be strongly variable.

\section{SUMMARY AND CONCLUSION}

In this work, we have presented the simulation results of the SIR event observed by both PSP and STEREO-A in September 2019. By coupling the energetic particle 


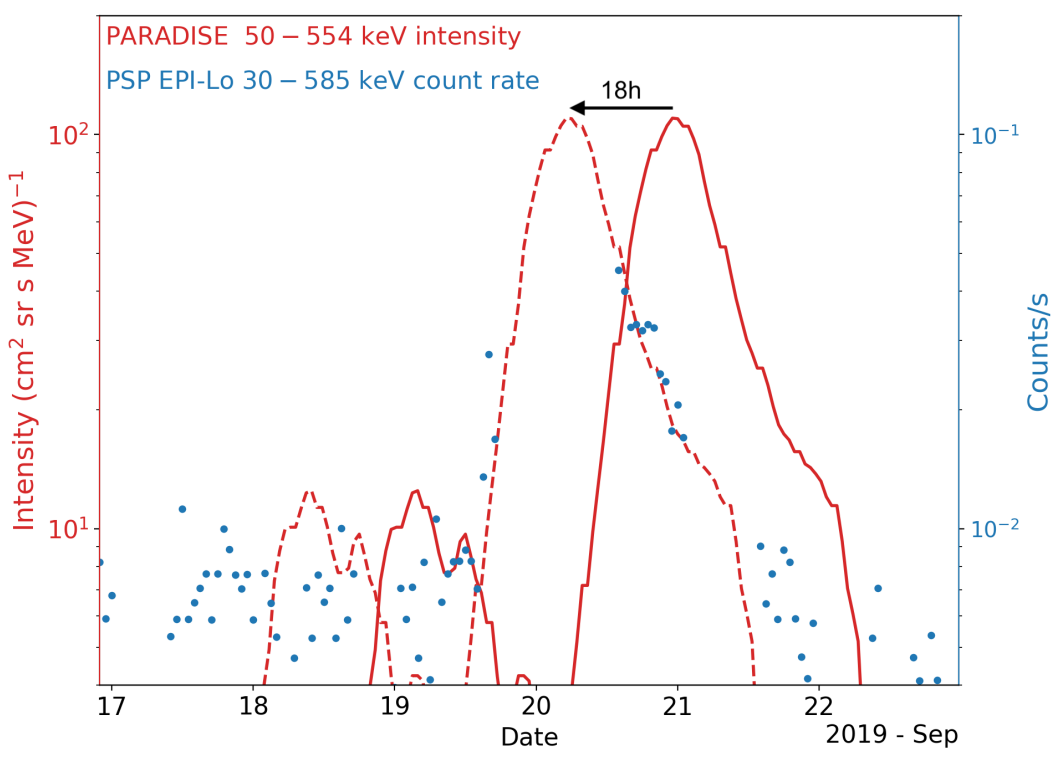

Figure 4. Count rates of 30-585 keV ions measured by the Time-of-Flight (ToF) system of the EPI-Lo instrument on PSP (blue dots) and the simulated 50-554 keV proton omnidirectional intensity (red solid line), along with the same simulated intensity but shifted $18 \mathrm{~h}$ in time (red dashed line)
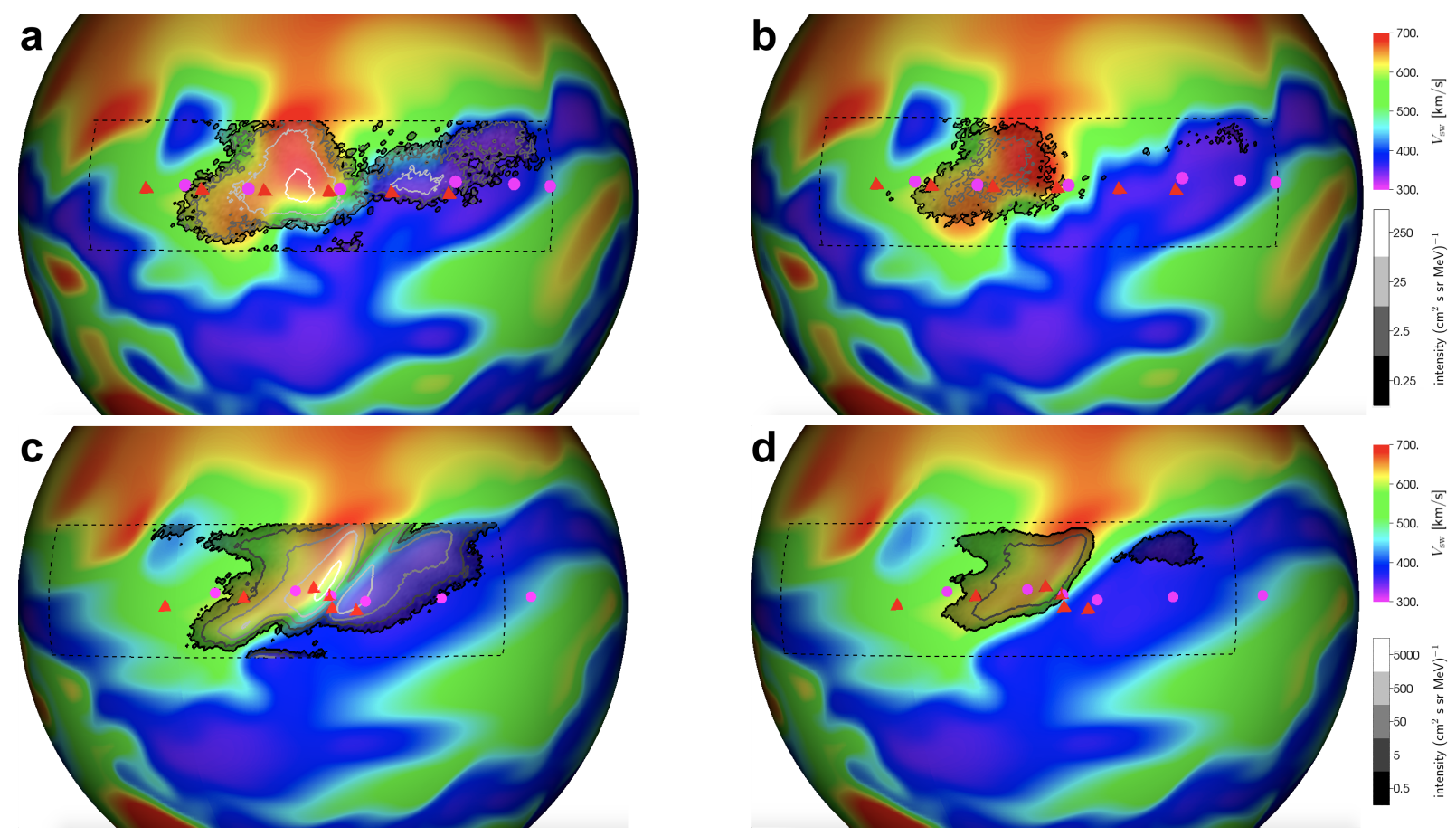

Figure 5. Rainbow-colour maps showing the solar wind speed at $r=0.5$ au (top row) and $r=1.5$ au (bottom row). The grey shades show the simulated $84.1-92.7 \mathrm{keV}$ (left) and $496.4-554.8 \mathrm{keV}$ (right) proton intensities. The symbols represent the magnetic connection of STEREO-A (pink dots) and PSP (red triangles) at a $24 \mathrm{~h}$ time cadence, ranging from 18 Sep 12:00 (rightmost symbols) to 23 Sep 12:00 (leftmost symbols). The dashed rectangles indicate the particle sampling domain of PARADISE. 
model PARADISE to the MHD model EUHFORIA, we were able to model a solar wind configuration and energetic particle enhancements that are in good agreement with the observations of STEREO-A and PSP. In our simulations, the energetic protons are accelerated selfconsistently at the compression waves bounding the SIR, assuming a seed population of $40 \mathrm{keV}$ protons. Such a seed population may originate from the solar wind suprathermal tail, especially near compression or shock waves where the solar wind gets heated adiabatically, thus producing more suprathermal protons (Prinsloo et al. 2019). Our simulations are consistent with the hypothesis that energetic particle enhancements near SIRs are mainly produced by the diffusive acceleration of solar wind suprathermal tail particles at the compression waves bounding SIRs. Low-energy particles $(\lesssim 500 \mathrm{keV})$ can already accelerate at small radial distances, before the compression waves have steepened sufficiently to accelerate $\mathrm{MeV}$ particles. Therefore, particles observed at a given heliospheric location may come from different regions, with the higher-energy particles being accelerated at larger helioradii. The stronger RCW (Fig. 2b) leads to a more efficient acceleration of high-energy particles near the trailing edge of the SIR. For these reasons, the simulated energy spectrum hardens toward the end of the SIR event, in agreement with the observations.

Earlier models predicted that the energy spectrum associated with SIR events would exhibit a turnover at low energies $(<0.5 \mathrm{MeV})$, because these ions undergo significant energy losses due to adiabatic deceleration as they propagate in the sunward direction (Fisk \& Lee 1980). However, such a turnover has not been observed in recent PSP observations (Desai et al. 2020; Joyce et al. 2020). Our simulations show that compression waves can accelerate suprathermal particles already within the orbit of Earth, which provides an explanation on why the energy spectra do not show a turnover at low energies.

In addition, the strong density pileup and magnetic field enhancement observed by PSP in front of the HSS indicates that our solar wind simulation likely underestimated the speed gradient at the leading edge of the HSS within the orbit of Earth. Steep speed gradients at small radial distances have been previously reported in Helios data (Schwenn 1990), suggesting a sharp transition between slow and fast solar wind sources. The compression waves associated with such sudden transitions might allow some particle acceleration to occur in close proximity to the Sun. Future PSP observations close to the Sun will allow for continued investigation on these particle acceleration processes.
No special turbulence conditions were assumed near the compression waves in our simulation. The good agreement between the energy spectrum of the simulation and the data suggests that any enhancements in the solar wind turbulence near the SIR might be of secondary importance to the acceleration process of suprathermal protons. The particle simulations did not include a momentum diffusion process, suggesting that stochastic acceleration is likely not a dominant acceleration process inside the SIR.

Furthermore, our simulations indicate that cross-field diffusion is likely not very efficient near and within the SIR. This is because the start and stop times of the simulated SIR event agree well with the observed ones. This agreement is lost when introducing a strong cross-field diffusion process, since it produces earlier start times and later stop times. In addition, a strong cross-field diffusion process would reshape the two-peak intensity profile seen in Fig. 3 into an intensity profile consisting of a single broad peak, which is not observed.

Finally, our simulations show that the threedimensional configuration of the solar wind streams can strongly modulate the energetic particle distributions, illustrating the importance of employing advanced three dimensional models when studying SIR events. Moreover, it indicates that small latitudinal differences between spacecraft can lead to very different energetic particle observations. In the future, data from SolO obtained outside the solar ecliptic will provide insight into latitudinal variations of the particle intensities associated with SIRs, and hence allow us to further constrain the scattering properties of energetic particles.

\section{ACKNOWLEDGEMENTS}

N. W. acknowledges funding from the Research Foundation - Flanders (FWO - Vlaanderen, fellowship no. 1184319 N). E. S. is supported by a PhD grant awarded by the Royal Observatory of Belgium. This project has received funding from the European Union's Horizon 2020 research and innovation programs under grant agreement No. 870405 (EUHFORIA 2.0). Computational resources and services used in this work were provided by the VSC (Flemish Supercomputer Centre), funded by the FWO and the Flemish GovernmentDepartment EWI. Support by ISSI and ISSI-BJ through the international team 469 is also acknowledged. A. A. acknowledges the support by the Spanish Ministerio de Ciencia e Innovación (MICINN) under grant PID2019-105510GB-C31 and through the "Center of Excellence María de Maeztu 2020-2023" award to the ICCUB (CEX2019-000918-M). D. L. acknowledges the support from the NASA-HGI grant NNX16AF73G 
and the NASA Program NNH17ZDA001N-LWS. The work was also carried out in the framework of the Finnish Centre of Excellence in Research of Sustainable Space (Academy of Finland Grant 312390). These results were also obtained in the framework of the projects C14/19/089 (C1 project Internal Funds KU Leuven), G.0D07.19N (FWO-Vlaanderen), and C 90347 (ESA Prodex). We acknowledge the use of STEREO SEPT data available at http://www2.physik.uni-kiel. de/stereo/data/sept/, and STEREO magnetic field and plasma data available at https://stereo-ssc.nascom. nasa.gov. We are also thankful to the Parker Solar Probe teams for providing IS $\odot$ IS/EPI-Lo and magnetic field and plasma data available at https://cdaweb.gsfc. nasa.gov. Parker Solar Probe was designed, built, and is now operated by the Johns Hopkins Applied Physics Laboratory as part of NASA's Living with a Star (LWS) program (contract NNN06AA01C). Support from the LWS management and technical team has played a critical role in the success of the Parker Solar Probe mission.

\section{APPENDIX}

\section{A. EUHFORIA SETUP}

The solar wind is modelled by using EUHFORIA, a physics-based coronal and heliospheric model designed for space weather research and prediction purposes (Pomoell \& Poedts 2018). The coronal module of EUHFORIA uses as input a synoptic magnetogram from the Global Oscillation Network Group (GONG) and applies the semi-empirical WangSheeley-Arge model (WSA; Arge et al. 2004) to provide the solar wind plasma and magnetic quantities at 0.1 au. These data are then utilised as the inner boundary conditions for the heliospheric module. The latter module solves the ideal MHD equations from 0.1 au up to a prescribed outer boundary, which is placed at 4 au in this work. The resolution of the spatial grid on which the MHD equations are solved is chosen to be $(\Delta r, \Delta \vartheta, \Delta \varphi)=\left(1.64 R_{\odot}, 1^{\circ}, 1^{\circ}\right)$, where $r, \vartheta$, and $\varphi$ denote the radial, latitudinal, and longitudinal coordinates, respectively.

In this study, the GONG magnetogram of 2019-09-18 06:14UT is used as input to the coronal module and the standard setup (Pomoell \& Poedts 2018) of EUHFORIA is used, except for the following three changes:

1. A constant value of $30 \mathrm{~km} / \mathrm{s}$ is added to the resulting WSA speed profile, and in addition, the speed profile is capped to be in the range $[340,700] \mathrm{km} / \mathrm{s}$ at $0.1 \mathrm{au}$.

2. The WSA solar wind speed map is rotated by $7.25^{\circ}$ instead of $10^{\circ}$, to account for rotation of the coronal magnetic field.

3. The magnetic field polarity is assumed to be everywhere positive at 0.1 au.

The first change is necessary to avoid a systematic underestimation of the slow and fast solar wind speed in the vicinity of the SIR. The second change is performed to ensure that the simulated arrival time of the HSS at STEREOA corresponds with the observed arrival time. The third change is done to avoid having a heliospheric current sheet (HCS) in the simulation, which is required by PARADISE as explained in the next paragraph.

\section{B. PARADISE SETUP}

The PARADISE (Wijsen 2020) model is used to calculate the temporal and spatial evolution of energetic particle populations that propagate through EUHFORIA solar wind configurations. This is achieved by solving the 5-dimensional focused transport equation (FTE; e.g., Roelof 1969; Skilling 1971; le Roux \& Webb 2012), which takes into account the effects of solar wind turbulence through a set of phase-space diffusion processes. The FTE utilised in PARADISE is expressed in guiding centre (GC) coordinates, and contains therefore the effects of GC drifts and cross-field diffusion (e.g., Zhang 2006; Wijsen 2020; Strauss et al. 2020). The GC drifts have, however, a negligible effect on the transport of the low-energetic protons studied in this work (Wijsen et al. 2020). The FTE accounts for particles' pitch-angle and momentum changes in compressional, shear, and accelerating solar wind flows (e.g., le Roux et al. 2007; Zank 2014). In particular, it takes into account the acceleration of particles in high-amplitude compression and shock waves (Giacalone et al. 2002; Wijsen et al. 2019a).

The FTE assumes that the solar wind varies on temporal and length scales that are larger than the energetic particles' gyroperiods and gryoradii, respectively. These assumptions may not be valid near the HCS (Burger et al. 1985; Wijsen 2020), which is why we do not include a HCS in our solar wind simulations. 


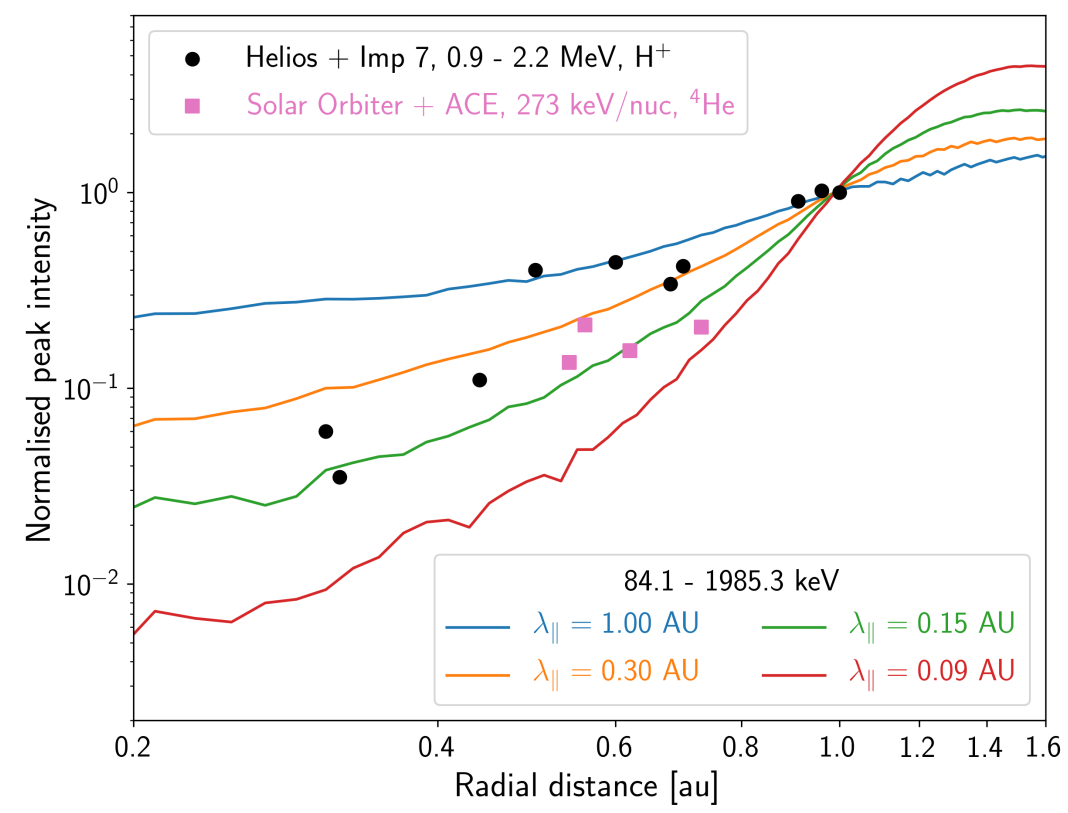

Figure 6. Simulated 94-1985 keV proton peak intensities as a function of heliocentric radial distance for different parallel mean free paths. The intensities have been normalised to their value at $1 \mathrm{au}$. The black dots and the grey squares correspond to SIR events observed by Helios (Van Hollebeke et al. 1978) and SolO (Allen et al. 2020c), respectively.

In this study, $40 \mathrm{keV}$ protons are injected in solar wind regions where the divergence of the solar wind velocity is negative, i.e., $\nabla \cdot \mathbf{V}_{\mathrm{sw}}<0$. As illustrated in Fig. 2, these regions include the RCW and the FCW bounding the SIR. In addition, we scale the injected particle distribution so that $f_{\mathrm{inj}}(t, \mathbf{x}) \propto\left|\nabla \cdot \mathbf{V}_{\mathrm{sw}}\right|$ (Prinsloo et al. 2019). This is done because a negative $\nabla \cdot \mathbf{V}_{\mathrm{sw}}$ gives a measure of local compression and therefore of adiabatic heating of the solar wind. In addition, regions where $\nabla \cdot \mathbf{V}_{\mathrm{sw}}<0$ are the locations where particles can gain energy by interacting with the converging scattering centres embedded in the solar wind flow.

The pseudo-particles are propagated for a total time period of 12 days in the solar wind generated by EUHFORIA. A reflective boundary condition is prescribed at the inner boundary $(0.1 \mathrm{au})$ and an absorbing boundary condition is prescribed at the outer boundary $(4 \mathrm{au})$. The particle injection distribution is assumed to be constant in time in the frame corotating with the Sun. This is achieved by convolving a Green's function solution of the FTE with a uniform time distribution. This is possible because the EUHFORIA solar wind solution is steady in the frame corotating with the Sun. The resulting proton distribution obtained after 12 days is steady-state in the corotating frame. This distribution is utilised to obtain the intensity-time profiles at PSP and STEREO-A presented in this work. Furthermore, the distribution is scaled such that the simulated intensity matches to the observed STEREO-A (peak) intensity in the 84.1-92.7 keV channel attained on 21 Sep 16:55 UT.

The pseudo-particles are sampled on a grid of phase-space volumes of size $2 \pi r^{2} \sin (\theta) \Delta \vartheta \Delta \varphi \Delta r \Delta E \Delta \mu$, where $E$ denotes the energy coordinate, $\mu$ the cosine of the pitch-angle, and $(r, \vartheta, \varphi)$ are spherical coordinates. We choose $\Delta r=0.02$ au, $\Delta \vartheta=\Delta \varphi=0.5^{\circ}$ and $\Delta \mu=0.1$. The energy $E$ is computed in the HEEQ reference frame upon sampling the pseudo-particles.

The FTE includes the effect of turbulence on the energetic particle transport through a set of diffusion processes in phase space. In this work, we include a cross-field diffusion process and a pitch-angle diffusion process. A constant perpendicular mean free path $\lambda_{\perp}=10^{-4}$ au is assumed to describe the cross-field diffusion coefficient as $\kappa_{\perp}=\frac{1}{3} v \lambda_{\perp}$. For the pitch-angle diffusion, we prescribe an anisotropic diffusion coefficient based on Quasi-Linear theory (Jokipii 1966), as implemented in PARADISE (Wijsen et al. 2019a). The magnitude of the pitch-angle diffusion is fixed by assuming a constant parallel mean free path $\lambda_{\|}$everywhere in the heliosphere.

Figure 6 shows the radial variation of the $84-1985 \mathrm{keV}$ proton peak intensities for different parallel mean free paths. The intensities are calculated as a six-hour average around the peak intensity seen by virtual spacecraft that are radially aligned and located at $2.3^{\circ}$ latitude (HEEQ). The intensities have been normalised to the value attained at $1 \mathrm{au}$. The dots give the $0.96-2.2 \mathrm{MeV}$ proton peak intensities determined by Van Hollebeke et al. (1978) of SIR 
events observed by the Helios spacecraft, relative to the peak intensities of the corresponding SIR event observed at 1 au by Imp 7 . The squares give the ${ }^{4} \mathrm{He}$ intensities determined by Allen et al. (2020c) of SIR events observed by SolO, relative to the intensities of the corresponding SIR events observed by the Advanced Composition Explorer (ACE; Stone et al. 1998) at 1 au. Despite the differences in ion species and energy ranges, a good match is obtained between the simulations and the observations. Data of the Sep 2019 SIR event discussed in this work are not included in the figure, since PSP did not observe the peak intensity. A mean free path of 1.0 au produces high intensities at small radial distances, since for such a large mean free path, the particles are only weakly coupled to the solar wind plasma. As a result, magnetic focusing is the dominant process in reversing the propagation direction of sunward streaming particles. In contrast, for the mean free path of $0.09 \mathrm{au}$, particles are more tightly coupled to the solar wind plasma, and consequently they are efficiently advected with the solar wind toward larger radial distances, resulting in particle intensities that decrease strongly with decreasing radial distance. Most observations included in Fig. 6 fit a parallel mean free path in the range 0.15 au $\lesssim \lambda_{\|} \lesssim 0.3$ au.

Each observational point presented in Fig. 6 is derived by combining the measurements of two spacecraft (HeliosIMP 7 or SolO-ACE) that were not necessarily located at exactly the same latitude. Figure 5 illustrates that, in our simulation, there is a clear variation of the particle intensities with latitude as a result of the underlying non-nominal solar wind conditions. Similarly, it was shown by e.g., Schwenn et al. (1978) and Jian et al. (2019) that spacecraft separated in latitude by just a few degrees can observe significant differences in the plasma properties of a single SIR. Such a latitudinal variation might be prevalent in SIR events and will contribute to the dispersion of the observational points presented in Fig. 6. In order to infer any general latitudinal intensity dependencies of SIR events, detailed studies of various SIR events seen at different latitudes are needed.

For the Sep 2019 SIR event discussed in this work, the best agreement between the simulated and observed eventintegrated energy spectrum at STEREO-A is attained for $\lambda_{\|}=0.3$ au. The latter mean free path also provides the best agreement between the observed and simulated start and stop times of the SIR event at both STEREO-A and PSP. Therefore, the simulation results presented in this work are for $\lambda_{\|}=0.3 \mathrm{au}$.

\section{REFERENCES}

Allen, R. C., Lario, D., Odstrcil, D., et al. 2020a, ApJS, 246, 36, doi: 10.3847/1538-4365/ab578f

Allen, R. C., Ho, G. C., Jian, L. K., et al. 2020b, A\&AForthcoming article, 18, doi: 10.1051/0004-6361/202039833

Allen, R. C., Mason, G. M., Rodriguez-Pacheco, J., et al. 2020c, A\&A, doi: 10.1051/0004-6361/202039870

Allen, R. C., Ho, G. C., Mason, G. M., et al. 2021, Geophysical Research Letters, n/a, e2020GL091376, doi: https://doi.org/10.1029/2020GL091376

Arge, C. N., Luhmann, J. G., Odstrcil, D., Schrijver, C. J., \& Li, Y. 2004, Journal of Atmospheric and Solar-Terrestrial Physics, 66, 1295, doi: 10.1016/j.jastp.2004.03.018

Barnes, C. W., \& Simpson, J. A. 1976, ApJL, 210, L91, doi: 10.1086/182311

Burger, R. A., Moraal, H., \& Webb, G. M. 1985, Ap\&SS, 116, 107, doi: 10.1007/BF00649278

Cohen, C. M. S., Christian, E. R., Cummings, A. C., et al. 2020, ApJS, 246, 20, doi: 10.3847/1538-4365/ab4c38

Desai, M. I., Mitchell, D. G., Szalay, J. R., et al. 2020, ApJS, 246, 56, doi: 10.3847/1538-4365/ab65ef

Fisk, L. A., \& Lee, M. A. 1980, ApJ, 237, 620, doi: 10.1086/157907
Fox, N. J., Velli, M. C., Bale, S. D., et al. 2016, SSRv, 204, 7, doi: 10.1007/s11214-015-0211-6

Giacalone, J., Jokipii, J. R., \& Kóta, J. 2002, ApJ, 573, 845, doi: 10.1086/340660

Gosling, J. T., Asbridge, J. R., Bame, S. J., \& Feldman, W. C. 1978, J. Geophys. Res.Space Phys., 83, 1401, doi: 10.1029/JA083iA04p01401

Gosling, J. T., \& Pizzo, V. J. 1999, SSRv, 89, 21, doi: 10.1023/A:1005291711900

Jian, L. K., Luhmann, J. G., Russell, C. T., \& Galvin, A. B. 2019, SoPh, 294, 31, doi: 10.1007/s11207-019-1416-8

Jokipii, J. R. 1966, ApJ, 146, 480, doi: 10.1086/148912

Joyce, C. J., McComas, D. J., Christian, E. R., et al. 2020, ApJS, 246, 41, doi: 10.3847/1538-4365/ab5948

le Roux, J. A., \& Webb, G. M. 2012, ApJ, 746, 104, doi: 10.1088/0004-637X/746/1/104

le Roux, J. A., Webb, G. M., Florinski, V., \& Zank, G. P. 2007, ApJ, 662, 350, doi: 10.1086/517601

Marshall, F. E., \& Stone, E. C. 1978, J. Geophys. Res., 83, 3289, doi: 10.1029/JA083iA07p03289

Mason, G. M., \& Sanderson, T. R. 1999, SSRv, 89, 77, doi: 10.1023/A:1005216516443

McComas, D. J., Alexander, N., Angold, N., et al. 2016, SSRv, 204, 187, doi: 10.1007/s11214-014-0059-1 
McComas, D. J., Christian, E. R., Cohen, C. M. S., et al. 2019, Nature, 576, 223, doi: 10.1038/s41586-019-1811-1

Müller, D., Marsden, R. G., St. Cyr, O. C., \& Gilbert, H. R. 2013, SoPh, 285, 25, doi: 10.1007/s11207-012-0085-7

Müller-Mellin, R., Böttcher, S., Falenski, J., et al. 2008, SSRv, 136, 363, doi: 10.1007/s11214-007-9204-4

Pomoell, J., \& Poedts, S. 2018, JSWSC, 8, A35, doi: $10.1051 /$ swsc/2018020

Porsche, H. 1975, Raumfahrtforschung, 19, 223

Prinsloo, P. L., Strauss, R. D., \& Le Roux, J. A. 2019, ApJ, 878, 144. https://arxiv.org/abs/1905.08458

Richardson, I. G. 1985, Planet. Space Sci., 33, 557, doi: 10.1016/0032-0633(85)90097-2

Richardson, I. G. 2018, Living Rev. Sol. Phys., 15, 1, doi: $10.1007 /$ s41116-017-0011-z

Roelof, E. C. 1969, in Lectures in High-Energy Astrophysics, ed. H. Ögelman \& J. R. Wayland, 111

Ruffolo, D. 1995, ApJ, 442, 861, doi: 10.1086/175489

Schwadron, N. A., Fisk, L. A., \& Gloeckler, G. 1996, Geophys. Res. Lett., 23, 2871, doi: 10.1029/96GL02833

Schwadron, N. A., Joyce, C. J., Aly, A., et al. 2020, A\&AForthcoming article, 14, doi: 10.1051/0004-6361/202039352

Schwenn, R. 1990, Large-Scale Structure of the Interplanetary Medium (Berlin, Heidelberg: Springer Berlin Heidelberg), 99-181, doi: 10.1007/978-3-642-75361-9_3
Schwenn, R., Montgomery, M. D., Rosenbauer, H., et al. 1978, J. Geophys. Res., 83, 1011, doi: 10.1029/JA083iA03p01011

Skilling, J. 1971, ApJ, 170, 265, doi: 10.1086/151210

Stone, E. C., Frandsen, A. M., Mewaldt, R. A., et al. 1998, SSRv, 86, 1, doi: 10.1023/A:1005082526237

Strauss, R. D., van den Berg, J. P., Steyn, P. J., et al. 2020, in Journal of Physics Conference Series, Vol. 1620, Journal of Physics Conference Series, 012021, doi: 10.1088/1742-6596/1620/1/012021

Van Hollebeke, M. A. I., McDonald, F. B., Trainor, J. H., \& von Rosenvinge, T. T. 1978, J. Geophys. Res., 83, 4723, doi: 10.1029/JA083iA10p04723

Wijsen, N. 2020, PhD thesis, KU Leuven (Belgium) and Univ. Barcelona (Spain). https://lirias.kuleuven.be/retrieve/573330

Wijsen, N., Aran, A., Pomoell, J., \& Poedts, S. 2019a, A\&A, 622, A28, doi: 10.1051/0004-6361/201833958

-. 2019b, A\&A, 624, A47, doi: 10.1051/0004-6361/201935139

—. 2019c, J. Phys.: Conf. Ser., 1332, 012018, doi: 10.1088/1742-6596/1332/1/012018

Wijsen, N., Aran, A., Sanahuja, B., Pomoell, J., \& Poedts, S. 2020, A\&A, 634, A82, doi: 10.1051/0004-6361/201937026

Zank, G. P. 2014, Transport Processes in Space Physics and Astrophysics, Lecture Notes in Physics (New York: Springer-Verlag), doi: 10.1007/978-1-4614-8480-6

Zhang, M. 2006, J. Geophys. Res.Space Phys., 111, A04208, doi: 10.1029/2005JA011323 